\title{
ON THE SENDOV CONJECTURE FOR SIXTH DEGREE POLYNOMIALS
}

\author{
JOHNNY E. BROWN
}

(Communicated by Clifford J. Earle, Jr.)

\begin{abstract}
The Sendov conjecture asserts that if $p(z)=\prod_{k=1}^{n}\left(z-z_{k}\right)$ is a polynomial with zeros $\left|z_{k}\right| \leq 1$, then each disk $\left|z-z_{k}\right| \leq 1,(1 \leq k \leq n)$ contains a zero of $p^{\prime}(z)$. This conjecture has been verified in general only for polynomials of degree $n=2,3,4,5$. If $p(z)$ is an extremal polynomial for this conjecture when $n=6$, it is known that if a zero $\left|z_{j}\right| \leq \lambda_{6}=0.626997 \ldots$ then $\left|z-z_{j}\right| \leq 1$ contains a zero of $p^{\prime}(z)$. (The conjecture for $n=6$ would be proved if $\lambda_{6}=1$.) It is shown that $\lambda_{6}$ can be improved to $\lambda_{6}=63 / 64=$ 0.984375 .
\end{abstract}

The well-known conjecture of Sendov [6, Problem 4.5] posed in 1962 states that if $p(z)=\prod_{k=1}^{n}\left(z-z_{k}\right),\left|z_{k}\right| \leq 1 \quad(1 \leq k \leq n)$, then each disk $\left|z-z_{k}\right| \leq 1$ $(1 \leq k \leq n)$ contains a zero of $p^{\prime}(z)$. Surprisingly this conjecture has been proved only for polynomials of degree $n \leq 5$ and in a few special cases [2-4, 9-15]. (See Marden [8] for an excellent expository article on this conjecture.) The case $n=5$ was proved in 1969 [9], while the general case for $n=6$ is still open.

The Sendov conjecture can be viewed as an extremal problem over a compact family of functions as follows. Let $\mathscr{P}_{n}$ denote the family of all monic polynomials of the form

$$
p(z)=\prod_{k=1}^{n}\left(z-z_{k}\right), \quad\left|z_{k}\right| \leq 1 \quad(1 \leq k \leq n) .
$$

Thus, by the classical Gauss-Lucas Theorem we have

$$
p^{\prime}(z)=n \prod_{j=1}^{n-1}\left(z-\zeta_{j}\right), \quad\left|\zeta_{j}\right| \leq 1 \quad(1 \leq j \leq n-1) .
$$

Define $I\left(z_{k}\right)=\min _{1 \leq j \leq n-1}\left|z_{k}-\zeta_{j}\right|, I(p)=\max _{1 \leq k \leq n} I\left(z_{k}\right)$, and $I\left(\mathscr{P}_{n}\right)=$ $\sup _{p \in \mathscr{P}_{n}} I(p)$. Phelps and Rodriguez [10] proved that there exists an extremal polynomial $p_{*}(z)=\prod_{k=1}^{n}\left(z-z_{k}^{*}\right) \in \mathscr{P}_{n}$ such that $I\left(p_{*}\right)=I\left(\mathscr{P}_{n}\right)$ (see Lemma

Received by the editors November 5, 1989; presented to the 96th Annual Meeting of the American Mathematical Society, January 1990.

1980 Mathematics Subject Classification (1985 Revision). Primary 30C15; Secondary 30C10. 
A). It thus suffices to prove the conjecture for $p_{*}$ i.e., show $I\left(z_{k}^{*}\right) \leq 1$ for $1 \leq$ $k \leq n$. They also proved a result which implies that if $n \geq 5$ and $z_{j}^{*}$, for some $j$, satisfies $\left|z_{j}^{*}\right| \leq \lambda_{n}$, where $\lambda_{n}$ is the unique root of $\left(1+x^{2}\right)(1+x)^{n-3}-n=0$, then $I\left(z_{j}^{*}\right) \leq 1$. In the special case $n=6$ their result gives $\lambda_{6}=0.626997 \ldots$. Of course the conjecture for $n=6$ would be proved if $\lambda_{6}=1$. The purpose of this paper is to improve the bound $\lambda_{6}$ :

Theorem. If $p_{*}(z)=\prod_{k=1}^{6}\left(z-z_{k}^{*}\right)$ is an extremal polynomial for $I\left(\mathscr{P}_{6}\right)$ and $\left|z_{j}^{*}\right| \leq 63 / 64$, for some $j=1,2, \ldots, 6$, then the disk $\left|z-z_{j}^{*}\right| \leq 1$ contains a zero of $p_{*}^{\prime}(z)$.

This result now reduces the conjecture for the case $n=6$ to considering the zeros of $p_{*}$ close to $|z|=1$. In this direction, we point out that Goodman, Rahman, and Ratti [5] have proved that if $z_{0}$ is a zero of $p(z)$ and $\left|z_{0}\right|=1$, then the disk $\left|z-z_{0} / 2\right| \leq \frac{1}{2}$ contains a zero of $p^{\prime}(z)$. Thus for boundary zeros a result stronger than the Sendov conjecture holds.

Our proof of the main result involves a blend of analytic and geometric ideas. The method presented here can be used to increase the bounds for $\lambda_{n}$ for all $n \geq 6$.

We make use of the following known results:

Lemma A (Phelps and Rodriguez [10]). There exists an extremal polynomial $p_{*} \in \mathscr{P}_{n}$ such that $I\left(p_{*}\right)=I\left(\mathscr{P}_{n}\right)$. Moreover, $p_{*}(z)$ has a zero on each subarc of $|z|=1$ of length $\pi$.

Lemma B (Bojanov, Rahman, and Szynal [2]). If $Q(z)$ is a monic polynomial of degree $n, Q(0)=0$, and $Q^{\prime}(z) \neq 0$ in $|z| \leq R$, then

$$
|Q(z)|>R^{n}-(R-\lambda)^{n} \text { for }|z|=\lambda \leq R \sin \pi / n \text {. }
$$

We also need the following estimates:

Lemma 1. Let $p(z)=(z-a) \prod_{k=1}^{n-1}\left(z-z_{k}\right) \in \mathscr{P}_{n}, 0<a<1$ and $p^{\prime}(z)=$ $n \prod_{j=1}^{n-1}\left(z-\zeta_{j}\right)$. If $I(a)>1$, then

$$
\left|\frac{\zeta_{j}-a}{a \zeta_{j}-1}\right|>\frac{1}{1+a-a^{2}} \quad j=1,2 \ldots, n-1 .
$$

Lemma 2. If $p(z)=(z-a) \prod_{k=1}^{n-1}\left(z-z_{k}\right)$ is an extremal polynomial for $I\left(\mathscr{P}_{n}\right)$, $0<a<1$ and $p^{\prime}(z)=n \prod_{j=1}^{n-1}\left(z-\zeta_{j}\right)$, then

$$
\prod_{j=1}^{n-1}\left|\frac{\zeta_{j}-a}{a \zeta_{j}-1}\right| \leq\left[n-(n-3) a-\frac{4 a^{2}}{1+a^{2}}\right]^{-1} \text {. }
$$

Lemma 3. Let $p(z)=(z-a) \prod_{k=1}^{5}\left(z-z_{k}\right)$ be an extremal polynomial for $I\left(\mathscr{P}_{6}\right)$.

(i) If $0 \leq a<\frac{2}{3}$, then $I(a) \leq 1$. 
(ii) If $\frac{2}{3} \leq a \leq \frac{63}{64}$ and $I(a)>1$, then there exists a zero $\zeta_{0}=a+\rho_{0} e^{i \theta_{0}}$ of $p^{\prime}(z)$ such that $\rho_{0}>1$ and

$$
\cos \theta_{0} \geq 1 / 25-a \text {. }
$$

Proof of Theorem. Suppose $p_{*}(z)=\prod_{k=1}^{6}\left(z-z_{k}^{*}\right)$ is an extremal polynomial for $I\left(\mathscr{P}_{6}\right)$ and suppose that $\left|z_{j}^{*}\right| \leq 63 / 64$ for some $j$. By a rotation, if necessary, we may suppose that $z_{j}^{*}=a, 0 \leq a \leq 63 / 64$. Hence

$$
p_{*}(z)=(z-a) \prod_{k=1}^{5}\left(z-z_{k}\right), \quad\left|z_{k}\right| \leq 1 \quad k=1,2, \ldots, 5 .
$$

Assume by way of contradiction that $I(a)>1$. By Lemma 3(i) we must then have $\frac{2}{3} \leq a \leq \frac{63}{64}$. Thus, making use of Lemma 3(ii) we can assert that there exists a critical point $\zeta_{0}=a+\rho_{0} e^{i \theta_{0}}$ with $\rho_{0}>1$ and $\cos \theta_{0} \geq 1 / 25-a$. It follows that

$$
\begin{aligned}
\left|\frac{\zeta_{0}-a}{a \zeta_{0}-1}\right| & \geq \frac{1}{\left[\left(1-a^{2}\right)^{2}+a^{2}-2 a\left(1-a^{2}\right) \cos \theta_{0}\right]^{1 / 2}} \\
& \geq \frac{1}{\left[\left(1-a^{2}\right)^{2}+a^{2}-2 a\left(1-a^{2}\right)(1 / 25-a)\right]^{1 / 2}} .
\end{aligned}
$$

Assuming as we may that $\left|\left(\zeta_{1}-a\right) /\left(a \zeta_{1}-1\right)\right| \leq\left|\left(\zeta_{j}-a\right) /\left(a \zeta_{j}-1\right)\right|$ for the critical points $\zeta_{j} \neq \zeta_{0}$, we apply Lemma 2 with $n=6$ to get

$$
\left|\frac{\zeta_{1}-a}{a \zeta_{1}-1}\right|^{4}\left|\frac{\zeta_{0}-a}{a \zeta_{0}-1}\right| \leq \prod_{j=1}^{5}\left|\frac{\zeta_{j}-a}{a \zeta_{j}-1}\right| \leq \frac{1}{6-3 a-4 a^{2} /\left(1+a^{2}\right)}
$$

Using (1) and (2) we obtain

$$
\left|\frac{\zeta_{1}-a}{a \zeta_{1}-1}\right|^{4} \leq \frac{\left[\left(1-a^{2}\right)^{2}+a^{2}-2 a\left(1-a^{2}\right)(1 / 25-a)\right]^{1 / 2}}{6-3 a-4 a^{2} /\left(1+a^{2}\right)} \equiv \phi(a) .
$$

Simple numerical calculations show that

$$
\phi(a) \leq \frac{1}{\left(1+a-a^{2}\right)^{4}} \quad \text { for } \frac{2}{3} \leq a \leq \frac{63}{64} .
$$

From (3) we conclude that $\left|\left(\zeta_{1}-a\right) /\left(a \zeta_{1}-1\right)\right| \leq 1 /\left(1+a-a^{2}\right)$, contradicting Lemma 1.

We now turn to the proofs of the lemmas.

Proof of Lemma 1. Assume by way of contradiction that $\left|\left(\zeta_{1}-a\right) /\left(a \zeta_{1}-1\right)\right| \leq$ $1 /\left(1+a-a^{2}\right)$. Hence we get $\left(\zeta_{1}-a\right) /\left(a \zeta_{1}-1\right)=r e^{i \theta}$, where $0 \leq r \leq$ $1 /\left(1+a-a^{2}\right)$. This gives $\zeta_{1}=\left(a-r e^{i \theta}\right) /\left(1-a r e^{i \theta}\right)$ and so

$$
\left|\zeta_{1}-a\right|=\frac{r\left(1-a^{2}\right)}{\left|1-a r e^{i \theta}\right|} \leq \frac{r\left(1-a^{2}\right)}{1-a r} \leq 1,
$$

which contradicts $I(a)>1$. 
Proof of Lemma 2. Suppose that $p(z)$ is extremal and

$$
\begin{gathered}
p(z)=(z-a) \prod_{k=1}^{n-1}\left(z-z_{k}\right), \quad\left|z_{k}\right| \leq 1 \quad(1 \leq k \leq n-1), \\
p^{\prime}(z)=n \prod_{j=1}^{n-1}\left(z-\zeta_{j}\right), \quad\left|\zeta_{j}\right| \leq 1 \quad(1 \leq j \leq n-1),
\end{gathered}
$$

and $0<a<1$. Let $z=T(w)=(w-a) /(a w-1)$ and note that

$$
p(T(w))=p_{0}(w)(a w-1)^{-n},
$$

where

$$
p_{0}(w)=A w\left(w^{n-1}+b_{n-1} w^{n-2}+\cdots+b_{1}\right) .
$$

The zeros of $p_{0}(w)$ are $0, w_{1}, w_{2}, \ldots, w_{n-1}$, where $w_{k}=T\left(z_{k}\right), 1 \leq k \leq$ $n-1$. Hence we see that

$$
b_{1}=(-1)^{n-1} \prod_{k=1}^{n-1} w_{k}
$$

and

$$
b_{n-1}=-\sum_{k=1}^{n-1} w_{k}
$$

Differentiating $p(T(w))$ gives

$$
\frac{d p(T(w))}{d w}=\frac{d p(T(w))}{d z} \cdot \frac{d z}{d w}=A_{\frac{1}{a}} p_{0}(w)\left[-a(a w-1)^{-n-1}\right],
$$

where

$$
A_{1 / a} p_{0}(w)=n p_{0}(w)+\left(\frac{1}{a}-w\right) p_{0}^{\prime}(w)=B \prod_{j=1}^{n-1}\left(w-\gamma_{j}\right)
$$

is the polar derivative of $p_{0}(w)$ with respect to $1 / a$ (see Marden [7, p. 44]). Thus we get

$$
p^{\prime}(T(w))=A_{1 / a} p_{0}(w)\left[-a(a w-1)^{-n-1} d w / d z\right]
$$

(where ' denotes differentiation with respect to $z$ ). It follows from (5), (8), and (9) that the zeros of $p^{\prime}(z)$ and $A_{1 / a} p_{0}(w)$ are related by

$$
\gamma_{j}=\frac{\zeta_{j}-a}{a \zeta_{j}-1}, \quad 1 \leq j \leq n-1
$$

Next, we see after a simple check that

$$
A_{1 / a} p_{0}(w)=B\left[w^{n-1}+\cdots+\left(\frac{b_{1}}{n+a b_{n-1}}\right)\right]=B \prod_{j=1}^{n-1}\left(w-\gamma_{j}\right)
$$


and so

$$
\prod_{j=1}^{n-1}\left|\gamma_{j}\right|=\left|\frac{b_{1}}{n+a b_{n-1}}\right|
$$

By Lemma $A$ there is a zero on each subarc of $|z|=1$ of length $\pi$. Hence without loss of generality

$$
\begin{cases}z_{n-1}=e^{i \theta_{0}}, & 0 \leq \theta_{0} \leq \pi / 2 \\ z_{n-2}=e^{i \theta_{1}}, & \theta_{0}+\pi \leq \theta_{1} \leq 2 \pi .\end{cases}
$$

(If $\operatorname{Im} z_{n-1}<0$, simply consider $\overline{p(\bar{z})}$.) It is easy to check that

$$
\begin{aligned}
\operatorname{Re}\left\{T\left(z_{n-1}\right)+T\left(z_{n-2}\right)\right\} & =\operatorname{Re}\left\{\left(\frac{e^{i \theta_{0}}-a}{a e^{i \theta_{0}}-1}\right)+\left(\frac{e^{i \theta_{1}}-a}{a e^{i \theta_{1}}-1}\right)\right\} \\
& \leq \frac{2 a-\left(1-a^{2}\right) \cos \theta_{0}}{\left(1+a^{2}\right)-2 a \cos \theta_{0}}+\frac{2 a+\left(1+a^{2}\right) \cos \theta_{0}}{\left(1+a^{2}\right)+2 a \cos \theta_{0}} \\
& =\frac{4 a\left(1+a^{2}\right)\left(1-\cos ^{2} \theta_{0}\right)}{\left(1+a^{2}\right)^{2}-4 a^{2} \cos ^{2} \theta_{0}} \leq \frac{4 a}{1+a^{2}} .
\end{aligned}
$$

Using (6), (7), (11), and the above inequality, we obtain

$$
\begin{aligned}
\prod_{j=1}^{n-1}\left|\frac{\zeta_{j}-a}{a \zeta_{j}-1}\right| & \leq \frac{1}{\left|n-a \sum_{k=1}^{n-1} T\left(z_{k}\right)\right|} \\
& \leq \frac{1}{n-a \sum_{k=1}^{n-1} \operatorname{Re} T\left(z_{k}\right)} \\
& \leq \frac{1}{n-4 a^{2} /\left(1+a^{2}\right)-a(n-3)}
\end{aligned}
$$

The proof of the lemma is complete.

Note that (11) immediately gives $\prod_{j=1}^{n-1}\left|\gamma_{j}\right| \leq 1 /(n-(n-1) a)$. However this estimate is not good enough for our purposes.

Proof of Lemma 3. Let $p(z)=(z-a) \prod_{k=1}^{5}\left(z-z_{k}\right)$ be extremal.

(i) $0 \leq a<\frac{2}{3}$. Clearly $I(0) \leq 1$ by the classical Gauss-Lucas Theorem. Next, from Lemma 2 with $n=6$ we see that

$$
\prod_{j=1}^{5}\left|\frac{\zeta_{j}-a}{a \zeta_{j}-1}\right| \leq \frac{1}{6-3 a-4 a^{2} /\left(1+a^{2}\right)}
$$

Assuming $\left|\left(\zeta_{1}-a\right) /\left(a \zeta_{1}-1\right)\right| \leq\left|\left(\zeta_{j}-a\right) /\left(a \zeta_{j}-1\right)\right|$ for $j=1,2, \ldots, 5$ we have from (12) and an easy calculation that

$$
\left|\frac{\zeta_{1}-a}{a \zeta_{1}-1}\right| \leq \frac{1}{\left(6-3 a-4 a^{2} /\left(1+a^{2}\right)\right)^{1 / 5}} \leq \frac{1}{1+a-a^{2}} .
$$

Thus, by Lemma 1 , we get $I(a) \leq 1$. 
(ii) $\frac{2}{3} \leq a \leq \frac{63}{64}$ and $I(a)>1$. For fixed $a$ we apply Lemma B with $Q(z)=p(z+a), n=6, R=1$, and $\lambda=1-(1-a)^{1 / 6}$ to conclude that

$$
|p(z)|>1-(1-\lambda)^{6}=a \geq|p(0)|
$$

for $|z-a|=\lambda=1-(1-a)^{1 / 6}$. Note that $\lambda \leq \frac{1}{2}$. Since $p^{\prime}(z) \neq 0$ in $|z-a| \leq 1$ and the degree of $p(z)$ is six, we see that by Alexander's Theorem (See Marden [7, p. 110, exercise 2]) that $p(z)$ is univalent in $|z-a| \leq \frac{1}{2}$. Thus from (13) we see that there exists a unique $z_{0}$ such that $\left|z_{0}-a\right| \leq \lambda$ with $p\left(z_{0}\right)=p(0)$. We may assume that $\operatorname{Im} z_{0} \geq 0$ (if not, consider $\overline{p(\bar{z})}$ ). Let $\Gamma_{0}$ be the perpendicular bisector of the segment from 0 to $z_{0}$, and let $H_{0}^{+}$ and $H_{0}^{-}$be the closed halfplanes bounded by $\Gamma_{0}$. By a variant of the GraceHeawood Theorem (see [1, Lemma 1] for example) we see that $p^{\prime}(z)$ has a zero in both $H_{0}^{+}$and $H_{0}^{-}$. Let $\omega_{0}=\mu_{0}\left(x_{0}, y_{0}\right)+i \nu_{0}\left(x_{0}, y_{0}\right)$ be the intersection of $\Gamma_{0}$ with the circle $|z-a|=1$ with $\nu_{0}>0$. It follows that $p^{\prime}(z)$ has a zero $\zeta_{0}=a+\rho_{0} e^{i \theta_{0}}$ with $\rho_{0}>1$ and $\cos \theta_{0} \geq \mu_{0}\left(x_{0}, y_{0}\right)-a$. (See Figure 1.) It suffices to prove that $\mu_{0}\left(x_{0}, y_{0}\right) \geq 1 / 25$.

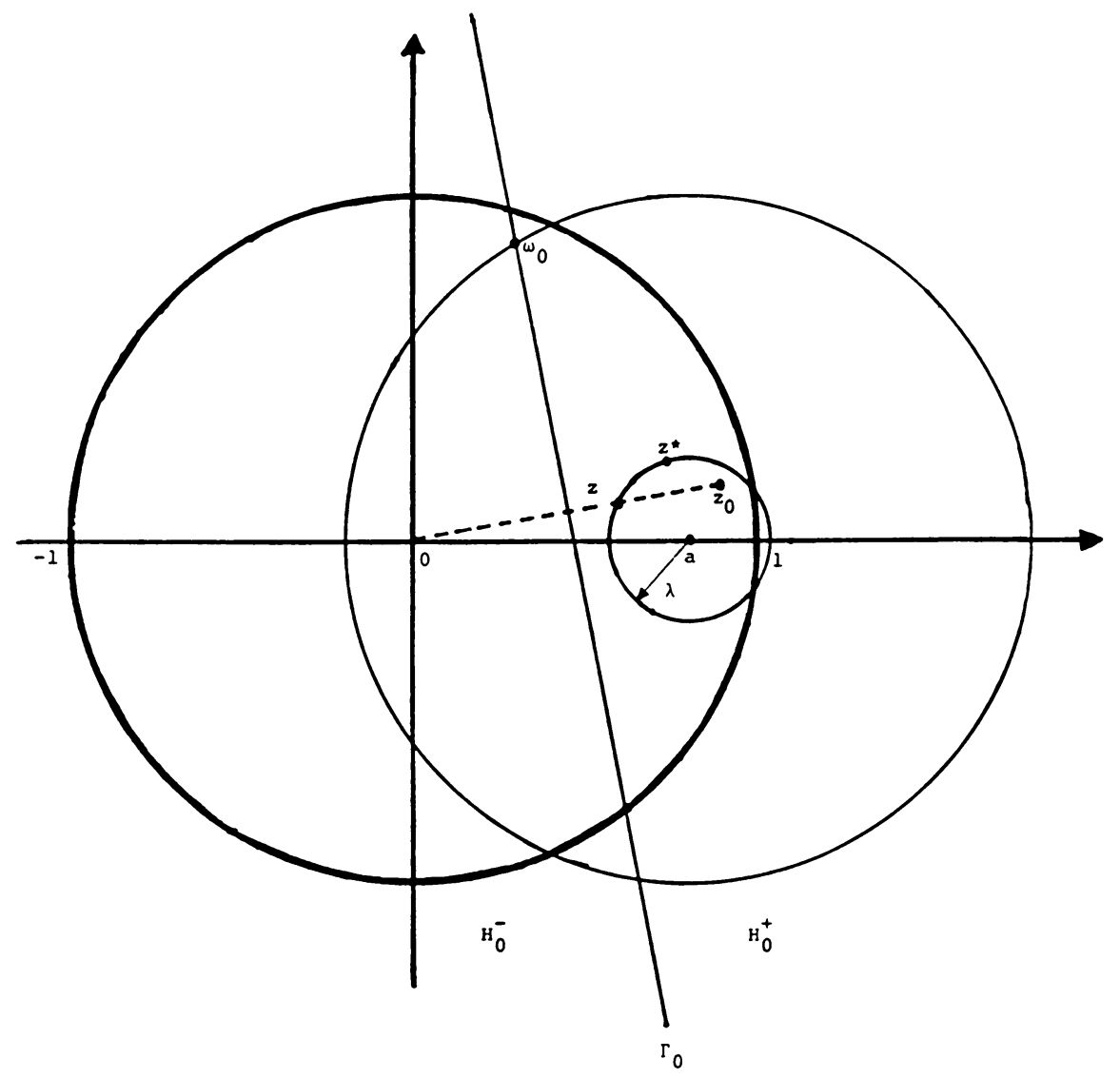

FIGURE 1 
Let $z^{*}=x^{*}+i y^{*}$ be the point on $|z-a|=\lambda$ such that the line through 0 and $z^{*}$ is tangent to $|z-a|=\lambda$ and $y^{*}>0$. Hence $x^{*}=\left(a^{2}-\lambda^{2}\right) / a$. If $z_{0}=r_{0} e^{i t_{0}}$ then by letting $z=r e^{i t_{0}}=x+i y \quad\left(r \leq r_{0}\right)$ be the point on $|z-a|=\lambda$ with $y>0$ and $x \leq x^{*}$, we conclude that $\mu_{0}\left(x_{0}, y_{0}\right) \geq \mu_{0}(x, y)$. Thus, it suffices to prove that $\mu_{0}(x, y) \geq 1 / 25$ where $(x-a)^{2}+y^{2}=\lambda^{2}$, $a-\lambda \leq x \leq x^{*}$, and $y \geq 0$.

A calculation shows that

$$
\mu_{0}(x, y)=\frac{x\left(\lambda^{2}+3 a^{2}\right)+2 a \beta-\sqrt{y^{2}\left(8 a x+4 \beta-\beta^{2}\right)}}{2(2 a x+\beta)},
$$

where $\beta=\lambda^{2}-a^{2}$. Observe that since $a-\lambda \leq x \leq x^{*}$ we get

$$
y^{2}=\lambda^{2}-(x-a)^{2} \leq 2 \lambda(x-a+\lambda) \text { and } 2 a x+\beta \leq-\beta .
$$

From (14) we conclude that

$$
\mu_{0}(x, y) \geq \frac{x\left(\lambda^{2}+3 a^{2}\right)+2 a \beta-\sqrt{2 \lambda(x-a+\lambda)\left(8 a x+4 \beta-\beta^{2}\right)}}{-2 \beta} \equiv \mu_{1} .
$$

It is enough to show that $\mu_{1} \geq 1 / 25$.

Now $\mu_{1} \geq 1 / 25$ if and only if

$$
F(x) \equiv c_{1} x^{2}+c_{2} x+c_{3} \geq 0, \quad a-\lambda \leq x \leq x^{*},
$$

where

$$
\begin{aligned}
& c_{1}=\left(\lambda^{2}+3 a^{2}\right)^{2}-16 a \lambda, \\
& c_{2}=2 \beta(2 a+0.08)\left(\lambda^{2}+3 a^{2}\right)-2 \lambda \beta(4-\beta)-16 a \lambda(\lambda-a), \\
& c_{3}=\beta^{2}(2 a+0.08)^{2}-2 \lambda \beta(4-\beta)(\lambda-a),
\end{aligned}
$$

$\left(\beta=\lambda^{2}-a^{2}\right)$. An easy check shows that $c_{1}>0$ and so (15) follows if $\Delta \equiv c_{2}^{2}-4 c_{1} c_{3}<0$. A brief calculation shows that $\Delta=4 \lambda(\lambda-a)^{2} \Delta_{0}$, where

$$
\begin{aligned}
\Delta_{0}= & {[(\lambda+a)(4-\beta)+8 a]\left[8 a \lambda+(\lambda+a)\left\{\lambda(4-\beta)-(4 a+0.16)\left(\lambda^{2}+3 a^{2}\right)\right\}\right] } \\
& +(\lambda+a)\left[16 a(\lambda+a)(2 a+0.08)^{2}+2(4-\beta)\left(\lambda^{2}+3 a^{2}\right)^{2}-32 a \lambda(4-\beta)\right] .
\end{aligned}
$$

Finally, a computation shows that $\Delta_{0}<0$ for $\frac{2}{3} \leq a \leq \frac{63}{64}$. (It can easily be checked numerically that $\Delta_{0}<-0.009$.) Thus (15) holds and hence $\mu_{0} \geq$ $1 / 25$.

The proof of the Sendov conjecture has been elusive for more than twentyfive years and only verified in a few special cases. The method of proof in the case $n=5$ does not seem to be useful for $n \geq 6$. It is not surprising to see the different ideas used to prove our results. 


\section{REFERENCES}

1. A. Aziz, On the zeros of a polynomial and its derivative, Bull. Austral. Math. Soc. 31 (1985), 245-255.

2. B. Bojanov, Q. I. Rahman, and J. Szynal, On a conjecture of Sendov about the critical points of a polynomial, Math. Z. 190 (1985), 281-285.

3. D. A. Brannan, On a conjecture of Ilieff, Proc. Cambridge Philos. Soc. 64 (1968), 83-85.

4. J. E. Brown, On the Ilieff-Sendov conjecture, Pacific J. Math. 135 (1988), 223-232.

5. A. W. Goodman, Q. I. Rahman, and J. Ratti, On the zeros of a polynomial and its derivative, Proc. Amer. Math. Soc. 21 (1969), 273-274.

6. W. K. Hayman, Research problems in function theory, Athlone Press, London, 1967, 56 pp.

7. M. Marden, Geometry of polynomials, Amer. Math. Soc. Surveys, no. 3, 1966.

8. 267-276.

9. A. Meir and A. Sharma, On Ilyeff's conjecture, Pacific J. Math. 31 (1969), 459-467.

10. D. Phelps and R. Rodriguez, Some properties of extremal polynomials for the Ilieff Conjecture, Kodai Math. Sem. Report 24 (1972), 172-175.

11. Z. Rubinstein, On a problem of Ilyeff, Pacific J. Math. 26 (1968), 159-161.

12. E. B. Saff and J. Twomey, A note on the location of critical points of polynomials, Proc. Amer. Math. Soc. 27 (1971), 303-308.

13. G. Schmeisser, Bermerkungen zu einer Vermutung von Ilieff, Math. Z. 111 (1969), 121-125.

14. __ Zur Lage der knitischen Punkte eines polynomes, Rend. Sem. Mat. Univ. Padova 46 (1971), 405-415.

15. __ On Ilieff's conjecture, Math. Z. 156 (1977), 165-173.

Department of Mathematics, Purdue University, West Lafayette, Indiana 47907 